\title{
Frequency-Independent Scattering for the Large Sphere
}

\author{
Wei E.I. Sha ${ }^{\# 1}$ and Weng Cho Chew ${ }^{\# 2}$ \\ \# Department of Electrical and Electronic Engineering, The University of Hong Kong \\ Pokfulam Road, Hong Kong \\ ${ }^{1}$ wshadeee.hku.hk ${ }^{2}$ wcchew@hku.hk
}

\begin{abstract}
The high frequency scattering of a scalar plane wave from an impenetrable sphere with the diameter of one thousand wavelength is treated by the saddle-point technique and the numerical steepest descent method. The far-field solution for the sphere is computed in the observation angle range of 0 to 180 degree. In particular, a novel numerical steepest descent method is proposed to overcome the breakdown of the traditional saddle-point technique in the forward region. Numerical results show that the CPU time for the far-field calculation is frequencyindependent with controllable error. This work can be used to benchmark future works in frequency-independent methods.

Index Terms-Saddle-Point Technique; Numerical Steepest Descent Method; Frequency Independent; High Frequency Scattering of Sphere.
\end{abstract}

\section{INTRODUCTION}

Mie series solution for scattering from an impenetrable sphere has been known for a long time [1][2]. Unfortunately, the Mie series will become useless at high frequencies due to its slow convergence. Hence, a large amount of summation terms must be used to obtain accurate results for both near-field and far-field analyses. The number of terms is proportional to $\beta=k a$ (where $k$ is the wavenumber and $a$ is the radius of sphere) for plane wave incidence and $k^{2} a^{2}$ for spherical or cylindrical wave incidence, which leads to expensive computational cost as the frequency increases. Although the geometrical optics method used in [3] is frequency-independent, it will breakdown for the forward scattering. Nussenzveig developed the uniform approximation method [4], but the author did not demonstrate the frequencyindependent results. In addition, the numerical solutions for the scattering by very large sphere could not be found in the literature. This work can be used to benchmark future works in frequency-independent methods.

In this paper, we calculate the far-field of the impenetrable sphere with the diameter of one thousand wavelength. In particular, the novel numerical steepest descent method (NSDM) is proposed to solve the forward scattering problem.

\section{THEORY}

\section{A. Backward Region}

For the far-field calculation, we have

$$
\Psi_{t}(r, \theta)=e^{i k z}+f_{s}(k, \theta) \frac{e^{i k r}}{r}
$$

where $\Psi_{t}$ is the total field, $e^{i k z}$ is the incident field, and $f_{s}(k, \theta)$ is the scattering amplitude, which can be approxi- mated as [5]

$$
f_{s}(k, \theta) \approx \frac{i}{k} \int_{\Gamma} \frac{H_{\lambda}^{(2)}(\beta)}{H_{\lambda}^{(1)}(\beta)} Q_{\lambda-1 / 2}^{(1)}(\cos \theta) \lambda d \lambda, \theta \gg 0
$$

where $\theta$ is the observation angle. The integration path $\Gamma$ crosses the real axis at the saddle-point $\bar{\lambda}=k p=\beta \cos (\theta / 2)$ with an angle of $-\pi / 4$. Using the asymptotic expansions (A-4) and (A-11) and using the saddle-point technique (SPT), we can get

$$
\begin{aligned}
f_{s}(k, \theta) & =-\frac{a}{2} \exp \left(-2 i \beta \sin \frac{\theta}{2}\right)\left(1+\frac{i}{2 \beta \sin ^{3} \frac{\theta}{2}}\right. \\
& \left.+\frac{2+3 \cos ^{2} \frac{\theta}{2}}{\left(2 \beta \sin ^{3} \frac{\theta}{2}\right)^{2}}+O\left[\left(2 \beta \sin ^{3} \frac{\theta}{2}\right)^{-3}\right]\right)
\end{aligned}
$$

According to geometrical optics, the first term of (3) represents the reflected wave. The high-order terms of (3) represent the corrections to geometrical optics corresponding to the WKB approximation. The expression agrees with the well-known conclusion in electromagnetics and optics that the backward radar cross section (RCS) of the large sphere is about $\pi a^{2}$.

\section{B. Forward Region}

However, as the observation angle $\theta$ approaches 0 , three things will happen: (1) the saddle-point will move towards the point $\lambda=\beta$; (2) the reflected ray and the incident ray meet at the same line called the focal line; (3) the Debye asymptotic expansions (A-4) are not available for $H_{\lambda}^{(1)}(\beta)$ and $H_{\lambda}^{(2)}(\beta)$. As a result, the expression of reflected wave (3) will diverge.

An alternative integral representation can be derived by the Poisson summation formula [5]

$$
\begin{aligned}
f_{s}(k, \theta) & \approx \frac{i}{k} \int_{0}^{\infty}[1-S(\lambda, k)] P_{\lambda-1 / 2}(\cos \theta) \lambda d \lambda \\
& -\frac{i}{k} \int_{i \infty}^{0} S(\lambda, k) P_{\lambda-1 / 2}(\cos \theta) \lambda d \lambda \\
& +\frac{2 i}{k} \int_{i \infty}^{0} \frac{e^{2 i \pi \lambda}}{1+e^{2 i \pi \lambda}} P_{\lambda-1 / 2}(\cos \theta) \lambda d \lambda
\end{aligned}
$$

where $S(\lambda, k)=-H_{\lambda}^{(2)}(\beta) / H_{\lambda}^{(1)}(\beta)$. In the neighborhood of the forward region, the main contribution to the integral arises from the neighborhood of $\lambda=\beta$. Thus, we can split the integral in the following form

$$
f_{s}(k, \theta) \approx f_{s a}+f_{s b}+f_{s c}
$$


where

$$
\begin{aligned}
f_{s a}+f_{s b} & =\frac{i}{k}\left(\int_{i \infty}^{0}+\int_{0}^{\beta}\right) \frac{H_{\lambda}^{(2)}(\beta)}{H_{\lambda}^{(1)}(\beta)} P_{\lambda-1 / 2}(\cos \theta) \lambda d \lambda \\
& +\frac{i}{k} \int_{\beta}^{\infty}\left(1+\frac{H_{\lambda}^{(2)}(\beta)}{H_{\lambda}^{(1)}(\beta)}\right) P_{\lambda-1 / 2}(\cos \theta) \lambda d \lambda
\end{aligned}
$$

and

$$
\begin{aligned}
f_{s c} & =\frac{i}{k} \int_{0}^{\beta} P_{\lambda-1 / 2}(\cos \theta) \lambda d \lambda \\
& +\frac{2 i}{k} \int_{i \infty}^{0} \frac{e^{2 i \pi \lambda}}{1+e^{2 i \pi \lambda}} P_{\lambda-1 / 2}(\cos \theta) \lambda d \lambda
\end{aligned}
$$

For $f_{s c}$, we can use the analytical method to evaluate its value [4][6], i.e.

$$
\begin{aligned}
f_{s c} & =i a\left(\frac{\theta}{\sin \theta}\right)^{1 / 2}\left[\frac{J_{1}(\beta \theta)}{\theta}-\frac{1}{8}\left(\frac{1}{\theta}-\cot \theta\right)\left(\frac{1-J_{0}(\beta \theta)}{\beta \theta}\right)\right. \\
& \left.+\frac{1}{24 \beta}\left(1+\frac{31}{120}\left(\frac{\theta}{2}\right)^{2}+\frac{53}{1008}\left(\frac{\theta}{2}\right)^{4}+\cdots\right)\right]
\end{aligned}
$$

For $f_{s a}$ and $f_{s b}$, we use the Fock transform [7] to find their steepest descent paths (SDPs). According to (A-5), in the neighborhood of $\lambda=\beta$, we have

$$
\frac{H_{\lambda}^{(2)}(\beta)}{H_{\lambda}^{(1)}(\beta)} \sim \frac{A i\left(x e^{-2 i \pi / 3}\right)}{A i\left(x e^{2 i \pi / 3}\right)} e^{2 i \pi / 3} \quad\left(\lambda<\beta,|\lambda-\beta| \lesssim \beta^{1 / 3}\right)
$$

$1+\frac{H_{\lambda}^{(2)}(\beta)}{H_{\lambda}^{(1)}(\beta)} \sim \frac{A i(x)}{A i\left(x e^{2 i \pi / 3}\right)} e^{i \pi / 3} \quad\left(\lambda>\beta,|\lambda-\beta| \lesssim \beta^{1 / 3}\right)$

where

$$
x=\gamma(\lambda-\beta), \gamma=\left(\frac{2}{\beta}\right)^{1 / 3}
$$

According to the asymptotic expansion of the Airy function (A-1), we know that the SDPs of $f_{s a}$ and $f_{s b}$ leave the real $x$ axis at the angles of $2 \pi / 3$ and 0 , i.e.

$$
\begin{gathered}
\lambda=\beta+\frac{\tilde{x}}{\gamma} e^{2 i \pi / 3}, \tilde{x}=x e^{-2 i \pi / 3} \\
\lambda=\beta+\frac{\tilde{x}}{\gamma}, \tilde{x}=x
\end{gathered}
$$

Along the SDPs, (9) and (10) are exponentially damped as the form $\exp \left(-\frac{4}{3} \tilde{x}^{3 / 2}\right)$. Hence the Gauss Legendre method [8] with the truncated integral interval can be employed to evaluate the integral. According to the numerical experiments, the integration intervals are set to $\tilde{x} \in[0,8]$ and $\tilde{x} \in[0,3.5]$, respectively. By (A-1) and (A-5), we find the approximated SDPs and set the integration intervals, but the integrands of $f_{s a}$ and $f_{s b}$ should be numerically evaluated by the uniform asymptotic expansions (A-2), (A-7) and (A-10).
When the observation angle moves away from 0 , the integrand of $f_{s b}$ decreases exponentially but it is not the case for $f_{s a}$. The integral $f_{s a}$ can be split as

$$
\begin{aligned}
f_{s a}=f_{s a}^{(1)}+f_{s a}^{(2)} & =\frac{i}{k} \int_{i \infty}^{\beta} \frac{H_{\lambda}^{(2)}(\beta)}{H_{\lambda}^{(1)}(\beta)} Q_{\lambda-1 / 2}^{(1)}(\cos \theta) \lambda d \lambda \\
& +\frac{i}{k} \int_{i \infty}^{\beta} \frac{H_{\lambda}^{(2)}(\beta)}{H_{\lambda}^{(1)}(\beta)} Q_{\lambda-1 / 2}^{(2)}(\cos \theta) \lambda d \lambda
\end{aligned}
$$

For $f_{s a}$, the term $Q_{\lambda-1 / 2}^{(2)}$ will decrease exponentially along the original SDP leaving the real $x$ axis at the angle of $2 \pi / 3$, but the term $Q_{\lambda-1 / 2}^{(1)}$ will increase exponentially along the SDP. According to (9), (12), (A-1), and (A-11), the integrand of $f_{s a}^{(1)}$ is dominated by the factor

$$
\exp \left(-\frac{4}{3} \tilde{x}^{3 / 2}+\frac{\sqrt{3}}{2} \frac{\theta}{\gamma} \tilde{x}\right) \exp \left(i \frac{\theta}{2 \gamma} \tilde{x}\right)
$$

The integrand has the peak $\tilde{x} \sim(\theta / \gamma)^{2}$ and oscillates as $\sim$ $\exp \left(i \frac{\theta}{\gamma} \tilde{x}\right)$. According to the numerical experiments, we need to change the SDP when $\theta>2 \gamma$. The integration path can be deformed as shown in Fig. 1. Obviously, the integration path will pass through the end point $x=0$ and the saddle-point $\bar{x}=\gamma(\bar{\lambda}-\beta)$.

When the observation angle $\theta$ is closed to $2 \gamma$, the integrand cannot be damped to a very small value as we expected. As $\theta$ increases, the vertical integration path from the end point $x=$ 0 can be seen as a "local" SDP. So the integration intervals for the saddle-point and the end point can be taken as, respectively,

$$
x \in\left[\max \left\{-C_{3},-\frac{C_{4}}{\cos (\pi / 4)}\right\}, C_{3}\right] e^{3 i \pi / 4}
$$

and

$$
x \in\left[0, \min \left\{C_{4}, C_{5}\right\}\right] e^{3 i \pi / 2}
$$

where $C_{3}=3.8(\theta / \gamma)^{1 / 2}, C_{4}=|\bar{x}|$, and $C_{5}=1.9(\theta / \gamma)^{1 / 2}$. As the frequency increases, the integration intervals will be slightly extended for a fixed observation angle. Fortunately, the available range of the forward region solution (3) will be extended to smaller angles also. Thus we need not increase the number of integration nodes as the frequency increases.

\section{NUMERICAL RESULTS}

We assume that $a=500$ and $k=2 \pi$. Fig. 2 shows the forward region solution by the NSDM. The solution is accurate between $0^{\circ}$ to $50^{\circ}$. Fig. 3 compares our solution with the original solution proposed in [4]. The number of the integration nodes is uniform for the two solutions. The original integration path proposed in [4] goes along the real $x$ axis from $x=0$ to $x=\bar{x}$ and the integrand will become very oscillatory as the observation angle increases. Fig. 4 shows the backward region solution by the SPT. The solution is accurate between $40^{\circ}$ to $180^{\circ}$. Hence, the NSDM solution and the SPT solution can overlap with each other. Fig. 5 shows the total CPU time for the far-field calculation as a function of the electrical size 


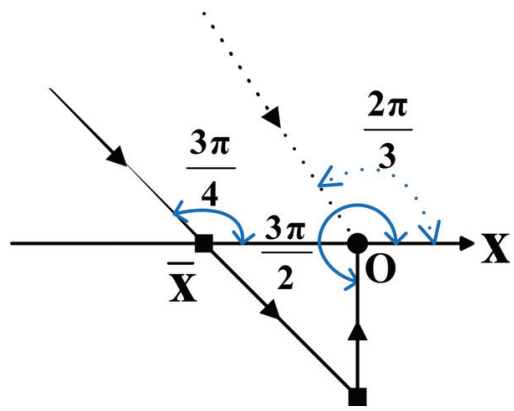

Fig. 1. The integration paths of $f_{s a}^{(1)}$. When the observation angle $\theta \leq 2 \gamma$, the path (dotted) leaves the real axis at the angle of $2 \pi / 3$ and goes to the end point $x=0$. When the observation angle $\theta>2 \gamma$, the revised path (solid) goes through the left saddle point related to the reflected wave and to the end point $x=0$. For the numerical implementation, we integrate $f_{s a}^{(1)}$ along the opposite direction and reverse the sign of the summation.

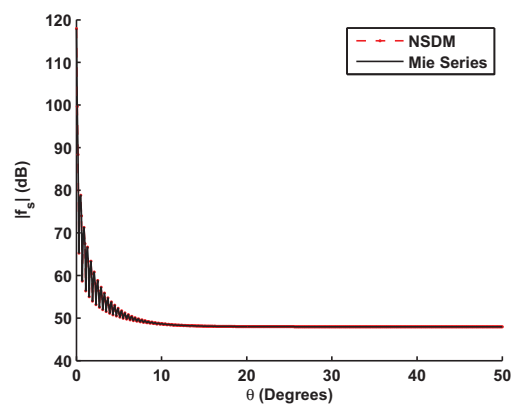

Fig. 2. The forward region solution of the scattering amplitude. The NSDM is adopted.

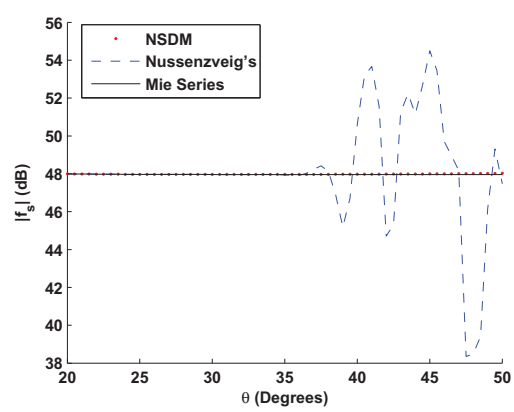

Fig. 3. The forward region solution of the scattering amplitude. The NSDM is proposed in this paper, and the original solution by Nussenzveig was proposed in [4].

of the radius. Here, we did not add any integration nodes. As the frequency increases, the SPT solution will become more accurate in the forward direction. Beacuse the analytical SPT is faster than the NSDM, the total CPU time is slightly decreased. Fig. 6 shows the relative two-norm error defined by

$$
E r r=\frac{\|N u m-M i e\|_{2}}{\|M i e\|_{2}}
$$

where Mie denotes the Mie series solution and Num denotes the solution by the NSDM and SPT. It can be seen that the error decreases as the frequency increases.

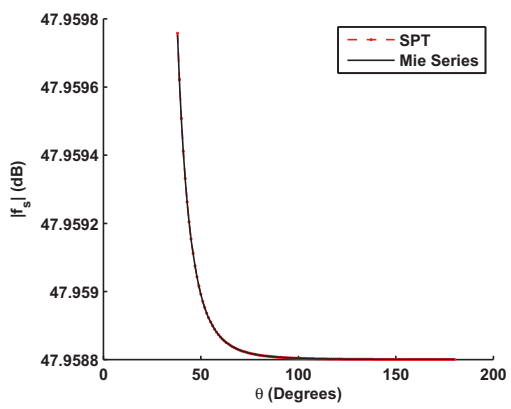

Fig. 4. The backward region solution of the scattering amplitude. The SPT is adopted.

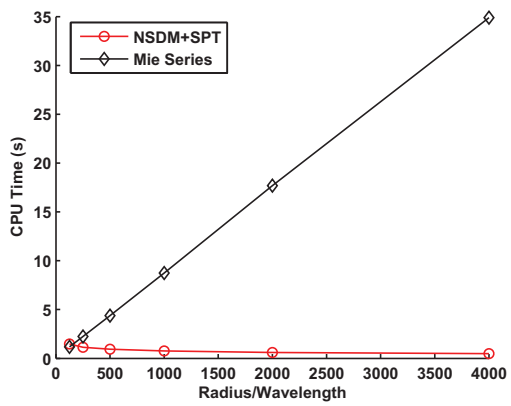

Fig. 5. The total CPU time for the far-field calculation. The NSDM and the SPT are used respectively for the forward region and the backward region.

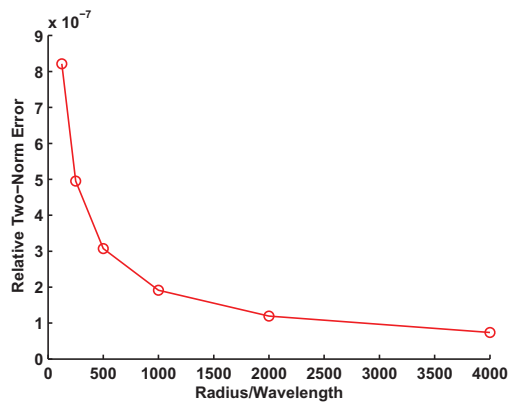

Fig. 6. The relative two-norm error for the far-field calculation. The Mie series solution is used as reference solution.

\section{CONCLUSiON}

The high frequency scattering of a scalar plane wave from a very large impenetrable sphere is computed. For the farfield calculation, the NSDM and the SPT are adopted for the forward and backward regions. The two solutions can overlap with each other. The total CPU time is frequency-independent and the numerical error can be controlled compared to the Mie series solution.

\section{APPENDIX}

In this Appendix, we will review the asymptotic expansions for special functions [5][6][9][10].

The asymptotic expansion of the Airy function is

$$
A i(z) \sim \frac{\exp \left(-\frac{2}{3} z^{3 / 2}\right)}{2 \sqrt{\pi} z^{1 / 4}},|z| \gg 1|\arg (\mathrm{z})|<\pi
$$


The uniform asymptotic expansion of the Hankel function is given by

$$
\begin{aligned}
& H_{\lambda}^{(1)}(\lambda z) \sim 2 e^{-\pi i / 3}\left(\frac{4 \xi}{1-z^{2}}\right)^{1 / 4}\left\{\frac{A i\left(e^{2 \pi i / 3} \lambda^{2 / 3} \xi\right)}{\lambda^{1 / 3}}+\right. \\
& \frac{e^{2 \pi i / 3} A i^{\prime}\left(e^{2 \pi i / 3} \lambda^{2 / 3} \xi\right)}{\lambda^{5 / 3}}\left[-\xi^{-1 / 2} \sum_{s=0}^{1} V_{s} \xi^{-3 s / 2} U_{1-s}\right. \\
& \left.\left.\left(\left(1-z^{2}\right)^{-1 / 2}\right)\right]\right\}
\end{aligned}
$$

where $V_{0}=1, V_{1}=5 / 48, U_{0}(t)=1, U_{1}(t)=\left(3 t-5 t^{3}\right) / 24$, and $A i$ and $A i^{\prime}$ are the Airy function and its derivative. The expansion for $H_{\lambda}^{(2)}(\lambda z)$ can be obtained by changing the sign of $i$ in (A-2). Here, we use the first two terms to obtain more accurate asymptotic representations. In (A-2), the important parameter $\xi$ should be treated carefully and the branch is chosen so that $\xi$ is real when $z$ is positive, i.e.

$$
\begin{aligned}
\frac{2}{3} \xi^{3 / 2} & =\ln \left(\frac{1+\sqrt{1-z^{2}}}{z}\right)-\sqrt{1-z^{2}}, \quad|z| \leq 1 \\
\frac{2}{3}(-\xi)^{3 / 2} & =\sqrt{z^{2}-1}-\arccos \left(\frac{1}{z}\right), \quad|z|>1
\end{aligned}
$$

The Debye expansions for the Hankel functions are given by

$$
\begin{aligned}
& H_{\lambda}^{(1,2)}(\rho) \sim(2 / \pi)^{1 / 2}\left(\rho^{2}-\lambda^{2}\right)^{-1 / 4} \exp \left\{ \pm i\left[\left(\rho^{2}-\lambda^{2}\right)^{1 / 2}\right.\right. \\
& \left.\left.-\lambda \cos ^{-1} \frac{\lambda}{\rho}-\pi / 4\right]\right\} \times\left[1 \mp \frac{i}{8\left(\rho^{2}-\lambda^{2}\right)^{1 / 2}} \times\left(1+\frac{5}{3}\right.\right. \\
& \left.\left.\times \frac{\lambda^{2}}{\rho^{2}-\lambda^{2}}\right)\right],-\rho<\lambda<\rho,|\lambda-\rho|>|\rho|^{1 / 3}
\end{aligned}
$$

The Debye expansions are not available when $|\lambda-\rho|$ becomes comparable with $|\lambda|^{1 / 3}$; we must employ the following expansions

$$
\begin{aligned}
H_{\lambda}^{(1,2)}(\rho) & \sim 2 \exp (\mp i \pi / 3)(2 / \lambda)^{1 / 3} \\
\cdot & A i\left[\exp ( \pm 2 i \pi / 3)(2 / \lambda)^{1 / 3}(\lambda-\rho)\right]
\end{aligned}
$$

Moreover, when $\rho \rightarrow \infty$, (A-4) reduces to the large augment expansion of the Hankel functions

$$
H_{\lambda}^{(1,2)}(\rho) \sim\left(\frac{2}{\pi \rho}\right)^{1 / 2} \exp \left[ \pm i\left(\rho-\lambda \frac{\pi}{2}-\frac{\pi}{4}\right)\right], \rho \rightarrow \infty
$$

The Szegö-Olver uniform asymptotic expansion for the Legendre function of the first kind is given by

$$
\begin{gathered}
P_{\lambda-1 / 2}(\cos \theta) \sim\left(\frac{\theta}{\sin \theta}\right)^{1 / 2}\left\{\left[1+\frac{1}{128 \lambda^{2}}\left(1-\frac{9}{\sin ^{2} \theta}\right.\right.\right. \\
\left.\left.\left.-6 \frac{\cot \theta}{\theta}+\frac{15}{\theta^{2}}\right)\right] J_{0}(\lambda \theta)-\frac{1}{8 \lambda}\left(\frac{1}{\theta}-\cot \theta\right) J_{1}(\lambda \theta)\right\}
\end{gathered}
$$

For $|\lambda| \theta \gg 1,(\mathrm{~A}-7)$ becomes

$$
P_{\lambda-1 / 2}(\cos \theta) \sim\left(\frac{2}{\pi \lambda \sin \theta}\right)^{1 / 2} \cdot\left[\cos \left(\lambda \theta-\frac{\pi}{4}\right)\right],|\lambda| \theta \gg 1
$$

The Legendre function of the first kind can be represented as

$$
P_{\lambda-1 / 2}(\cos \theta)=Q_{\lambda-1 / 2}^{(1)}(\cos \theta)+Q_{\lambda-1 / 2}^{(2)}(\cos \theta)
$$

The uniform asymptotic expansions for $Q_{\lambda-1 / 2}^{(1,2)}(\cos \theta)$ are

$$
\begin{aligned}
& Q_{\lambda-1 / 2}^{(1,2)}(\cos \theta) \sim\left(\frac{\theta}{\sin \theta}\right)^{1 / 2}\left\{\frac { 1 } { 2 } \left[1+\frac{1}{128 \lambda^{2}}\left(1-\frac{9}{\sin ^{2} \theta}\right.\right.\right. \\
& \left.\left.\left.-6 \frac{\cot \theta}{\theta}+\frac{15}{\theta^{2}}\right)\right] H_{0}^{(2,1)}(\lambda \theta)-\frac{1}{16 \lambda}\left(\frac{1}{\theta}-\cot \theta\right) H_{1}^{(2,1)}(\lambda \theta)\right\}
\end{aligned}
$$

For $|\lambda| \theta \gg 1,(\mathrm{~A}-10)$ becomes

$$
Q_{\lambda-1 / 2}^{(1,2)}(\cos \theta) \sim \frac{\exp [\mp i(\lambda \theta-\pi / 4)]}{(2 \pi \lambda \sin \theta)^{1 / 2}},|\lambda| \theta \gg 1
$$

\section{REFERENCES}

[1] G. Mie, "Beiträge zur Optik trüber Medien, speziell kolloidaler Metallösungen," Annalen der Physik, vol. 25, pp. 377-445, 1908.

[2] J. A. Kong, Electromagnetic Wave Theory. New York: WileyInterscience, 1990.

[3] J. Shim and H. T. Kim, "An asymptotic solution of EM backscattering from a conducting sphere coated with a composite material," IEEE Transactions on Antennas and Propagation, vol. 52, pp. 1465-1472, Jun 2004.

[4] H. M. Nussenzveig, "Uniform approximation in scattering by spheres," Journal of Physics A: Mathematical and General, vol. 21, pp. 81-109, Jan 1988.

[5] H. M. Nussenzveig, "High-frequency scattering by an impenetrable sphere," Annals of Physics, vol. 34, pp. 23-95, 1965.

[6] M. Abramowitz and I. A. Stegun, Handbook of Mathematical Functions with Formulas, Graphs, and Mathematical Tables. New York: Dover Publications, Inc., 1970.

[7] V. A. Fock, "Diffraction of radio waves around the earth's surface," Journal of Physics-USSR, vol. 9, pp. 255-266, 1945.

[8] W. H. Press, S. A. Teukolsky, W. T. Vetterling, and B. P. Flannery, Numerical Recipes: The Art of Scientific Computing: Cambridge University Press, 2007.

[9] R. Paknys, "Evaluation of Hankel functions with complex argument and complex order," IEEE Transactions on Antennas and Propagation, vol. 40, pp. 569-578, May 1992.

[10] F. W. J. Olver, "The asymptotic expansion of Bessel functions of large order," Philosophical Transactions of the Royal Society of London. Series A, Mathematical and Physical Sciences, vol. 247, pp. 328-368, Dec 1954. 